\title{
Personality Characteristics Associated with Attitudes towards the COVID-19 Virus*
}

\author{
Apeksha Srivastava ${ }^{1}$, Frederick L. Coolidge ${ }^{2}$ \\ ${ }^{1}$ Humanities and Social Sciences, Indian Institute of Technology Gandhinagar, Palaj, Gandhinagar, Gujarat, India \\ ${ }^{2}$ Psychology Department, 1420 Austin Bluffs Parkway, University of Colorado, Colorado Springs, Colorado, USA \\ Email: fcoolidg@uccs.edu
}

How to cite this paper: Srivastava, A., \& Coolidge, F. L. (2021). Personality Characteristics Associated with Attitudes towards the COVID-19 Virus. Psychology, 12, 1217-1229. https://doi.org/10.4236/psych.2021.128076

Received: July 6, 2021

Accepted: August 7, 2021

Published: August 10, 2021

Copyright (C) 2021 by author(s) and Scientific Research Publishing Inc. This work is licensed under the Creative Commons Attribution International License (CC BY 4.0).

http://creativecommons.org/licenses/by/4.0/ (c) (i) Open Access

\begin{abstract}
Background: We examined the presence of five maladaptive personality trait domains and 14 personality disorder traits from $D S M-5$ with regard to attitudes towards the COVID-19 virus, i.e., whether viewed as a serious threat (COVID-19 Group) or not (Denier/Minimizer Group). Method: 146 undergraduate and graduate students in India participated online to answer the questionnaire. Ages ranged from 18 to 33 years old (100 men, 46 women). Results: Consistent with hypotheses, the COVID-19 Group $(n=66)$ scored significantly higher on the negative affectivity and detachment trait domains and higher on the avoidant and depressive personality disorder traits than the Denier/Minimizer Group $(n=77)$. Contrary to hypotheses, the COVID-19 Group scored significantly higher on the disinhibition trait domain and Borderline personality disorder scale. Importantly, 20.3\% of the COVID-19 Group endorsed the current suicidal ideation item compared to only $11.7 \%$ of the Denier/Minimizer Group. While a greater percentage of the COVID-19 Group adhered to the pandemic precautions, a majority of participants in both groups were compliant with social distancing, handwashing/sanitizing, and face-masking. Conclusion: Gaining an understanding of these personality variations might assist in establishing efficient public health actions to mitigate health threats.
\end{abstract}

\section{Keywords}

COVID-19, Personality Trait Domains, DSM-5, Suicidal Ideation, Pandemic Precautions

\section{Introduction}

The purpose of the present study was to examine a broad array of personality ${ }^{\star}$ Personality characteristics. 
characteristics with regard to compliance with the COVID-19 virus management mandates and also to assess attitudes towards the virus, i.e., whether the virus should be viewed as a serious threat or not. The novel coronavirus disease 2019 (better known as COVID-19) emerged towards the end of 2019 and spread rapidly worldwide (Gralinski \& Menachery, 2020; Petrosillo et al., 2020). This infection was declared a global pandemic by the World Health Organization on 11th March 2020, with 118,000 confirmed cases accompanied by over 4200 deaths in 114 countries (WHO, 2020a). As of 16th July 2021, it engulfed 223 countries with a confirmed case count of more than 188,332,972 and 4,063,453 deaths (WHO, 2020a). This pandemic has forced people throughout the world to make drastic changes in their lifestyles.

Because of the lack of a cure for COVID-19 and dramatically effective treatments, countries have implemented some common general practices to help prevent the spread of the virus. These include covering of mouth and nose, frequent handwashing, maintaining proper hygiene, social (physical) distancing (people at least six feet apart), isolation of suspected and confirmed cases, quarantining of persons who were in contact with confirmed cases, closure of educational institutions and workplaces, stay-at-home recommendations, cancellation of mass gatherings, and mandatory lockdowns in residential areas, cities, and countries (WHO, 2020a). Nevertheless, these mitigation measures have led to some adverse psychological effects and health impacts on people. For example, isolation has been observed to contribute towards increased loneliness and stress, the risk factors for the development and progression of anxiety and depression (Matias et al., 2020). A recent study by Miguel et al. (2021) took the novel approach of examining not the psychological repercussions of the pandemic, but the relationship of personality characteristics to compliance with COVID-19 virus interventions. Thus, the present study examined specific personality characteristics that might differentiate between those who complied with the virus containment measures and those who would not. Further, we analyzed the personality characteristics of those who viewed the virus as a serious threat and those who did not.

It has been observed that compliance with COVID-19 mitigation practices and behaviors vary greatly among people, and there is a recognition that these practices and behaviors are linked to personality traits to a considerable extent. Lam (2021) identified some emerging "COVID-19 personality types," which appear to lead to the increased politicization of the pandemic. Some of these personality types included those who downplayed the viral threat, those who resisted containment measures in the name of personal freedom, those who placed blame upon other people and countries for the virus' spread, and those who took the virus seriously along with the suggested containment measures. One major limitation of the Lam study was that these types are not aligned with conventional psychological diagnoses, such as described in the Diagnostic and Statistical Manual of Mental Disorders (DSM-5; American Psychiatric Association, 2013) or in the World Health Organization's International Classification of Dis- 
eases (ICD-11; 2019).

Oosterhoff and Palmer (2020) suggested the association of social-trust responsibility (empathy) with greater compliance to preventive measures, less hoarding of supplies, and proper maintenance of hygiene. Carvalho et al. (2020) demonstrated that extroversion was directly proportional to more difficulties in adhering to containment measures. Murphy et al. (2021) found people with lower levels of conscientiousness were also more likely to be hesitant or resistant to be vaccinated. O'Connell et al. (2021) showed that people with lower levels of agreeableness, higher levels of Machiavellianism (manipulative behavior), psychopathy, and narcissism were less likely to comply with COVID-19 prevention measures. Miguel et al. (2021), as noted earlier, found that the antisocial traits of deceitfulness, callousness, risk-taking, and lower levels of empathy were directly correlated with lower compliance to COVID-19 restrictions.

However, the Miguel et al. (2021) study did not examine other maladaptive personality traits or other types of personality disorders, according to DSM-5 or $I C D-11$. Thus, the present research examined the presence of five categories of maladaptive personality trait domains from DSM-5. These five trait domains possessed individual facets within them. They were Negative affectivity (emotional lability, anxiousness, separation insecurity, submissiveness, hostility, perseveration); Detachment (withdrawal, intimacy avoidance, anhedonia, depressivity, restricted affectivity, suspiciousness); Antagonism (manipulativeness, deceitfulness, grandiosity, attention-seeking, callousness, hostility); Disinhibition (irresponsibility, impulsivity, distractibility, risk-taking, rigid perfectionism), and Psychoticism (unusual beliefs and experiences, eccentricity, cognitive and perceptual dysregulation). The present study also examined 14 personality disorder traits from $D S M-I I I-R, D S M-I V$, and $D S M-5$ : avoidant, dependent, depressive, obsessive-compulsive, paranoid, schizoid, antisocial, borderline, histrionic, narcissistic, passive-aggressive, sadistic, self-defeating, and schizotypal.

In the present study, all of these traits were assessed as a function of whether people viewed the virus as a serious threat or not. Thus, participants were divided into two groups: the COVID-19 Group (people who thought COVID-19 was a highly dangerous, deadly, and contagious) and the Denier/Minimizer Group (people who thought COVID-19 was mostly a hoax or an exaggeration used for political/other purposes, or COVID-19 was no more deadly or contagious than the common flu). The COVID-19 Group was hypothesized to be higher on the Negative Affectivity and Detachment maladaptive trait domain scales, while the Denier/Minimizer Group was hypothesized to be higher on the Antagonism, Disinhibition, and Psychoticism domain scales. The COVID-19 Group was also hypothesized to be higher on the Avoidant, Dependent, Depressive, Obsessive-compulsive, Paranoid, and Schizoid personality disorder scales, while the Denier/Minimizer Group was hypothesized to be higher on the Antisocial, Borderline, Histrionic, Narcissistic, Passive-aggressive, Sadistic, Self-defeating, and Schizotypal personality disorder scales. To the authors' knowledge, this is the first on- 
line study focused on analyzing the personality traits and changes associated with attitudes towards the COVID-19 virus in an Indian population sample.

\section{Method}

\subsection{Participants}

The present study was a sample of convenience, consisting of 146 bachelors, masters, and doctoral students from the Indian Institute of Technology (IIT) Gandhinagar, India. There were 100 men and 46 women, with the mean age = 21.46 years old, age range $=18$ to 33 years old. There were 88 undergraduates, 40 students in master's degree programs, and 18 doctoral students. The recruitment process was carried out through e-mails comprising an introductory letter along with the online Google Form of the questionnaire to be filled. There was compensation of $100 \mathrm{INR}$, and the participants needed to complete the study to receive the reward as noted in the informed consent. Successful submission of the completed Google Form was the proof of consent of the participants. This project was approved by the IITGN Institute Ethics Committee, and online data collection was carried out from 25th March 2021 to 29th March 2021.

\subsection{Measures}

The participants were asked to answer 137 questions (five demographic questions, four general COVID-19 related questions, and 128 personality-trait items). The personality-trait items were answered on a Likert-type scale: 1) strongly false, 2) more false than true, 3) more true than false, 4) strongly true.

\subsubsection{Questions about Attitudes towards COVID-19}

There were four general COVID-19 related questions to examine attitudinal differences between people who take the virus as a serious threat and those who do not. The first question was used to divide the participants into either the COVID-19 Group or the Denier/Minimizer Group. The other three questions were used from the Miguel et al. (2021) study to assess adherence to preventive/containment measures. They were: 1) Is it necessary to avoid approaching people until the COVID-19 situation is controlled? 2) Is it necessary to frequently wash hands and/or use alcohol sanitizer until the COVID-19 situation is controlled? 3) Is it necessary to use a facemask to cover nose and mouth?

\subsubsection{The Personality Trait Questions}

As noted previously, $D S M-5$ contains five maladaptive personality trait domains with 25 specific facets. Because each facet contained various descriptions, 58 personality-trait items were generated by the PIs based on their clinical relevance to the study.

\subsubsection{The Short-Form of the Coolidge Axis II Inventory (SCATI)}

The SCATI (Coolidge, 2019) is a self-report, 70-item inventory, which assesses 14 personality disorders, according to $D S M-5$ (10 personality disorders), $D S M-I V-T R$ (passive-aggressive and depressive), and DSM-III-R (self-defeating 
and sadistic). The SCATI has been shown to be reliable and valid in a variety of clinical and non-clinical settings (e.g., Coolidge et al., 2010; Fiala et al., 2020; Furnham \& MacRae, 2020).

\subsection{Procedure}

Data collection was carried out through online mode in the form of a self-report questionnaire. The participatory email circulated among the IIT Gandhinagar student community informed the participants of the principal investigators (PIs) involved, key information about the nature of the study, and the amount of time that it would possibly take to complete the same. The participants were informed that: 1) there would be no known risks involved by participating in the study and that there is no deception involved, 2) no individual feedback would be given during the study, although they could contact the PIs for group results in approximately six months from the completion of the study, 3) their participation would be completely voluntary and anonymous, and 4) that they may quit the study at any point without any consequences (other than forfeiting the payment). The average time to complete the questionnaire was about 30 mins. All participants were assigned an arbitrary ID. The electronic data was gathered, analyzed (using the SPSS V27 software), and maintained on a password-protected computer to ensure privacy and confidentiality of the information.

\section{Results}

\subsection{Internal Reliability of Measures}

In the present study, the internal reliabilities (Cronbach's $\alpha$ ) for the five maladaptive personality domain traits were as follows: Negative Affectivity (14 items) $\alpha=.89$; Detachment (15 items) $\alpha=.89$; Antagonism (7 items) $\alpha=.77$; Disinhibition (13 items) $\alpha=.87$; and Psychoticism ( 7 items) $\alpha=.81$. Thus, the internal reliabilities of the five domain traits may be considered ranging from fair to good.

The internal reliabilities (Cronbach's $\alpha$ ) for the SCATI personality disorder scales were as follows: Antisocial (5 items) $\alpha=.61$; Avoidant (5 items) $\alpha=.62$; Borderline (5 items) $\alpha=.56$; Dependent (5 items) $\alpha=.64$; Depressive (5 items) $\alpha$ $=.78$; Histrionic (5 items) $\alpha=.75$; Narcissistic (5 items) $\alpha=.63$; Obsessive-Compulsive (5 items) $\alpha=.55$; Paranoid (5 items) $\alpha=.66$; Passive-Aggressive (5 items) $\alpha=.62$; Sadistic (5 items) $\alpha=.72$; Self-Defeating (5 items) $\alpha=.63$; Schizotypal (5 items) $\alpha=.69$; and Schizoid (5 items) $\alpha=.52$. Given that Cronbach's $\alpha$ is strongly influenced by the number of items, the internal reliability of SCATI personality disorder scales may be considered acceptable or better.

\subsection{Hypothesis Testing for the Five Maladaptive Personality Trait Domain Scales}

To test the hypotheses, the participants were divided into two groups based on their attitudes towards the COVID-19 virus: the COVID-19 Group $(n=69)$ believed that the virus was highly dangerous, deadly, and contagious, and the 
Denier/Minimizer Group $(n=77)$ believed that it was mostly a hoax or an exaggeration used for political/other purposes, or it was no more deadly or contagious than the common flu. The first five hypotheses for the domain scales were tested by independent $t$-tests between the COVID-19 Group and the Denier/Minimizer Group. The results of these $t$-tests are presented in Table 1. There was support for the initial hypotheses that the COVID-19 group would be significantly higher on Negative Affectivity and Detachment scales. However, contrary to the original hypotheses, the COVID-19 group was higher on the other three scales, Antagonism, Disinhibition, and Psychoticism, although only the Disinhibition scale was significant.

\subsection{Hypothesis Testing for the 14 SCATI Personality Disorder Scales}

With regard to the initial hypotheses for the 14 personality disorder scales, the results of these t-tests are also presented in Table 1. Despite hypothesizing that there would be significant differences between the two groups on all 14 scales, there were only three scales that were significant. Two of these scales, Avoidant and Depressive, were significantly higher in the COVID-19 Group as hypothesized. Contrary to the original hypothesis, the COVID-19 Group was also significantly higher on the Borderline scale.

\subsection{Suicidal Ideation}

It is important to note that the detachment domain has an item that measures current suicidal ideation. It was found that 20.3\% of the COVID-19 Group endorsed this item but only $11.7 \%$ of the Denier/Minimizer Group endorsed it. Although this represents a 1.7 times greater rate of suicidal ideation in the COVID-19 Group than the Denier/Minimizer Group, it did not reach statistical significance, $\chi^{2}(1, \mathrm{n}=146)=2.03, p=.15$.

\subsection{Compliance to COVID-19 Management Measures}

The present study also included three questions from Miguel et al. (2021), which assessed compliance with three COVID-19 management measures: social (physical) distancing, frequent handwashing or using alcohol based sanitizer, and use of facemasks. Therefore, the two groups were assessed for their compliance with these three measures. A greater percentage of the COVID-19 Group adhered to these precautions, and a vast majority of participants in both groups were compliant with all three measures. However, there was only one significant difference between the two groups, which was in regard to frequent handwashing or using alcohol based sanitizer. 98.6\% of the COVID-19 Group endorsed it while $84.4 \%$ of the Denier/Minimizer Group endorsed it, $\chi^{2}(1, \mathrm{n}=146)=8.96, p$ $=.003 .94 .2 \%$ of the COVID-19 Group endorsed social distancing as compared to $85.7 \%$ of the Denier/Minimizer Group, $\chi^{2}(1, \mathrm{n}=146)=2.84, p=.09$. With regard to use of facemasks, $98.6 \%$ of the COVID-19 Group endorsed it in comparison to $92.2 \%$ of the Denier/Minimizer Group, $\chi^{2}(1, \mathrm{n}=146)=3.21, p=.07$. 
Table 1. (a) The $t$-test results between the COVID-19 and denier/minimizer groups for the five maladaptive personality trait domain scales; (b) The $t$-test results between the COVID-19 and denier/minimizer groups for the 14 SCATI personality disorder scales.

(a)

\begin{tabular}{|c|c|c|c|c|}
\hline Trait Domain & Groups & Mean $(S D)$ & $t$ value, $p$ level & $\begin{array}{l}\text { Effect Size } \\
\text { (Cohen's } d \text { ) }\end{array}$ \\
\hline \multirow[b]{2}{*}{ Negative Affectivity } & COVID-19 & $35.23(9.12)$ & \multirow[b]{2}{*}{$3.28, p=.001$} & \multirow[b]{2}{*}{.55 (medium) } \\
\hline & Denier/Minimizer & $30.45(8.13)$ & & \\
\hline \multirow{2}{*}{ Detachment } & COVID-19 & $42.42(11.06)$ & \multirow{2}{*}{$1.99, p=.048$} & \multirow{2}{*}{.33 (small) } \\
\hline & Denier/Minimizer & $38.92(10.15)$ & & \\
\hline \multirow{2}{*}{ Antagonism } & COVID-19 & $16.88(4.53)$ & \multirow{2}{*}{$1.73, p=.087$} & \multirow{2}{*}{.29 (small) } \\
\hline & Denier/Minimizer & $15.55(4.62)$ & & \\
\hline \multirow{2}{*}{ Disinhibition } & COVID-19 & $33.80(7.76)$ & \multirow{2}{*}{$2.44, p=.016$} & \multirow{2}{*}{.41 (small) } \\
\hline & Denier/Minimizer & $30.49(8.23)$ & & \\
\hline \multirow{2}{*}{ Psychoticism } & COVID-19 & $15.93(4.83)$ & \multirow{2}{*}{$1.38, p=.171$} & \multirow{2}{*}{.23 (small) } \\
\hline & Denier/Minimizer & $14.82(4.71)$ & & \\
\hline
\end{tabular}

(b)

\begin{tabular}{|c|c|c|c|c|}
\hline Disorder & Groups & Mean $(S D)$ & $t$ value, $p$ level & $\begin{array}{l}\text { Effect Size } \\
\text { (Cohen's } d \text { ) }\end{array}$ \\
\hline \multirow{2}{*}{ Antisocial } & COVID-19 & & \multirow{2}{*}{$1.09, p=.280$} & .18 \\
\hline & Denier/Minimizer & $8.92(2.68)$ & & (less than small) \\
\hline \multirow[b]{2}{*}{ Avoidant } & COVID-19 & $12.93(2.84)$ & \multirow[b]{2}{*}{$2.95, p=.004$} & \multirow[b]{2}{*}{$.50($ medium $)$} \\
\hline & Denier/Minimizer & $11.42(3.19)$ & & \\
\hline \multirow{2}{*}{ Borderline } & COVID-19 & $10.57(3.10)$ & \multirow{2}{*}{$2.18, p=.031$} & \multirow{2}{*}{.37 (small) } \\
\hline & Denier/Minimizer & $9.48(2.80)$ & & \\
\hline \multirow{2}{*}{ Dependent } & COVID-19 & $10.67(3.20)$ & \multirow{2}{*}{$1.25, p=.213$} & \multirow{2}{*}{.21 (small) } \\
\hline & Denier/Minimizer & $10.01(2.97)$ & & \\
\hline \multirow{2}{*}{ Depressive } & COVID-19 & $12.41(3.75)$ & \multirow{2}{*}{$3.28, p=.001$} & \multirow{2}{*}{.55 (medium) } \\
\hline & Denier/Minimizer & $10.37(3.61)$ & & \\
\hline \multirow{2}{*}{ Histrionic } & COVID-19 & $11.22(3.41)$ & \multirow{2}{*}{$.06, p=.955$} & \multirow{2}{*}{$\begin{array}{c}.01 \\
\text { (less than small) }\end{array}$} \\
\hline & Denier/Minimizer & $11.18(3.78)$ & & \\
\hline \multirow{2}{*}{ Narcissistic } & COVID-19 & $12.65(3.06)$ & \multirow{2}{*}{$.02, p=.986$} & \multirow{2}{*}{$\begin{array}{c}.003 \\
\text { (less than small) }\end{array}$} \\
\hline & Denier/Minimizer & $12.66(3.47)$ & & \\
\hline \multirow{2}{*}{ Obsessive-Compulsive } & COVID-19 & $12.30(2.86)$ & \multirow{2}{*}{$.51, p=.610$} & \multirow{2}{*}{$\begin{array}{c}.09 \\
\text { (less than small) }\end{array}$} \\
\hline & Denier/Minimizer & $12.06(2.88)$ & & \\
\hline \multirow{2}{*}{ Paranoid } & COVID-19 & $11.30(3.16)$ & \multirow{2}{*}{$1.03, p=.305$} & \multirow{2}{*}{$\begin{array}{c}.17 \\
\text { (less than small) }\end{array}$} \\
\hline & Denier/Minimizer & $10.73(3.41)$ & & \\
\hline \multirow{2}{*}{ Passive Aggressive } & COVID-19 & $11.16(2.90)$ & \multirow{2}{*}{$.82, p=.414$} & \multirow{2}{*}{$\begin{array}{c}.14 \\
\text { (less than small) }\end{array}$} \\
\hline & Denier/Minimizer & $10.73(3.25)$ & & \\
\hline
\end{tabular}


Continued

\begin{tabular}{ccccc}
\hline \multirow{2}{*}{ Sadistic } & COVID-19 & $8.39(3.32)$ & $.40, p=.693$ & $\begin{array}{c}.07 \\
\text { (less than small) }\end{array}$ \\
& Denier/Minimizer & $8.18(2.89)$ & & .22 (small) \\
Self-Defeating & COVID-19 & $11.39(3.07)$ & $1.31, p=.192$ & .08 \\
& Denier/Minimizer & $10.70(3.13)$ & & (less than small) \\
\hline \multirow{2}{*}{ Schizotypal } & COVID-19 & $9.20(3.36)$ & $.50, p=.618$ & .18 \\
& Denier/Minimizer & $9.48(3.18)$ & & (less than small) \\
\hline \multirow{2}{*}{ Schizoid } & COVID-19 & $10.26(2.88)$ & $1.05, p=.294$ & \\
& Denier/Minimizer & $9.76(2.74)$ & &
\end{tabular}

\section{Discussion}

The COVID-19 pandemic has wreaked havoc across the world since the end of 2019. In the initial absence of an effective vaccine, the virus could only be managed by isolation and quarantining of the positive/suspected cases, social (physical) distancing, wearing of facemasks, regular hand-washing, and using sanitizers. Studies have shown a considerable part of people's compliance with these measures to be dependent on their personality traits (Carvalho et al., 2020; Oosterhoff \& Palmer 2020; Miguel et al., 2021; Murphy et al., 2021; O'Connell et al., 2021). Therefore, the purpose of the present study was to explore various personality characteristics in an Indian student sample between those who took the virus as a serious threat and those who did not.

\subsection{Hypotheses Regarding the Five Maladaptive Personality Trait Domain Scales}

With regard to the initial hypotheses for the five maladaptive trait domains, there was statistical support for the hypotheses that negative affectivity and detachment would be higher in the COVID-19 Group than in the Denier/Minimizer Group. However, contrary to expectations, disinhibition was also significantly higher in the COVID-19 Group than the other group. There were no significant differences between the two groups on the antagonism and psychoticism domains, although the Denier/Minimizer Group was hypothesized to be higher on both domains.

It is intuitively reasonable that the group that took the virus seriously experienced more symptoms associated with the domain of negative affectivity, as it is a measure of increased guilt, depression, mood instability, irritability, anxiety, worry, nervousness, and apprehension about the future. The same is true of the detachment domain as it is a measure of the avoidance of social contacts, moving away from typical social interactions, pessimism about the future, and a greater awareness of how compliance measures can interfere and disrupt typical relationships.

Although contrary to the hypothesis, the COVID-19 Group scored significantly higher on the Disinhibition scale. However, upon further investigation of 
the individual items on this scale, it was found that more than half of the items (eight out of 13) assessed distractibility, risk taking behaviors, and impulsivity. Thus, it does make sense retrospectively that someone who takes the virus seriously would be more distracted, more prone to take risks because of their greater frustrations with COVID-19 mitigation measures, and more impulsive in their daily activities and in their short- and long-term goals than someone who either denied or minimized the virus' influence.

Interestingly and contrary to the initial hypotheses, the Denier/Minimizer Group was not significantly elevated on the antagonism and psychoticism domains. In fact, the COVID-19 Group trended higher on both domains although not significantly and with a small effect size. Upon subsequent investigation of the individual items, it appeared that the antagonism domain was not a pure measure of hostility and enmity but more a measure of self-concern rather than a concern for others' needs and feelings. Further, one of the seven individual items was a measure of guilt or remorse about relationship transgressions, which might actually be considered a trait not associated with antagonistic individuals. This same phenomenon may also be true of a greater psychoticism trend in the COVID-19 Group, as this domain was not defined by the classic symptoms of hallucinations and delusions but was defined by altered or unusual experiences of reality, disconnection from one's environment, and less continuity among one's thoughts, ideas, self-identity, and memories. Again in retrospect, it appears entirely reasonable that the COVID-19 Group might be elevated on such items.

\subsection{Hypotheses Regarding the 14 SCATI Personality Disorder Scales}

With regard to the initial hypotheses for the 14 SCATI personality disorder scales, there was statistical support that Avoidant and Depressive scales would be higher in the COVID-19 Group than in the Denier/Minimizer Group. Again, this finding is intuitively reasonable as all traits of avoidance revolve around a reluctance or resistance to social interactions. The same is true of the traits on the Depressive personality scale, which revolve around pessimism about the future and excessive self-doubt.

However, contrary to the original hypothesis, the COVID-19 Group was also significantly higher on the Borderline scale. An inspection of the latter traits showed a concern with abandonment by others, self-doubt, and suicidal threats or gestures. Again it is entirely reasonable that a group who considered the virus as a serious threat would react to social distancing measures, which profoundly interrupts both intimate and social relationships.

Contrary to the initial hypotheses, there were no significant differences between the two groups on the Antisocial, Dependent, Histrionic, Narcissistic, Obsessive-Compulsive, Paranoid, Passive-Aggressive, Sadistic, Self-Defeating, Schizotypal, and Schizoid scales. It is of particular interest that the two groups responded in a similar manner on the Antisocial scale, which is a contrast to the finding of the Miguel et al. (2021) study stating that higher levels of antisocial 
traits (Callousness, Deceitfulness, and Risk-taking) were directly associated with lower compliance to COVID-19 containment measures. It is possible that this difference in findings may be due to the focus of the SCATI antisocial items upon classic psychopathic symptoms, such as conning others, deceitfulness, and taking unfair advantage of others. Thus, it would not be surprising that the two groups would not be significantly different on such a classic measure of antisocial behavior. It is also important to note that the SCATI did not contain antisocial items directly measuring callousness and risk-taking. Future studies should take into account a much wider variety of symptoms associated with antisocial behavior than the much narrower five criteria of the antisocial SCATI scale.

\subsection{Suicidal Ideation}

Alarmingly, it was found that $20.3 \%$ of the COVID-19 Group endorsed a suicidal ideation item on the Detachment scale, while only $11.7 \%$ of the Denier/Minimizer Group did so. Although this difference was not statistically significant, it still represented a 1.7 times greater prevalence of suicidal ideation in the COVID-19 Group. Since it is well established that greater rates of suicidal ideation are associated with greater rates of suicide (e.g., Brezo et al., 2006), it would behoove those officials who deal with the virus to be aware of some of the serious secondary consequences (i.e., psychological and social) such as an increase in suicidal ideation and suicidal behaviors.

\subsection{Compliance to Virus Management Measures}

With regard to compliance to COVID-19 management measures, it is important to note that although a greater percentage of the COVID-19 Group adhered to these precautions (with only one significant difference with regard to handwashing/sanitizing), a vast majority of participants in both groups were compliant with all three measures. Across all three measures, the COVID-19 Group averaged 97\% compliance, while the Denier/Minimizer Group averaged 87\% compliance. This finding may be attributed to the nature of the sample, i.e., a group of students at IIT Gandhinagar. This Institute like many others throughout India strongly promoted these mitigation measures of isolation and quarantining of suspected/infected cases, social distancing, handwashing/sanitizing, and the wearing of facemasks. In fact, officials at IIT Gandhinagar imposed fines on anyone on its campus who did not wear a facemask in public. These steps explain why a vast majority of participants in both the COVID-19 Group and the Denier/Minimizer were compliant with all three measures.

\section{Limitations and Concluding Remarks}

There are some methodological limitations in the present study. First, it was focused on a restricted sample of convenience of IIT students with advanced educations, who were obviously not representative of the Indian population, which limited the study's generalization. Thus, future studies should include a much 
wider range of levels of education among participants. Further, analyses were not conducted as a function of gender, since the sample consisted of about $70 \%$ men, and some studies had shown that women tend to be more compliant than men in general (e.g., Coolidge et al., 2004). Second, the data was collected online in the form of self-reports, which inherently might be influenced by factors such as social desirability of one's answers in spite of the anonymity promised to the participants. Third, the data was gathered over a single time period, and it might be beneficial if future studies assessed compliance and personality traits over longer periods of time, particularly if certain personality traits such as risk taking become more florid as social distancing and quarantining increase with increasing surges of the virus. Fourth, it became clear that a wider variety of symptoms and traits associated with personality disorders should be included in future studies, as the SCATI was shown to be useful, but it had a narrower focus than some other personality disorder measures.

The present study represented an initial foray into examining the personality characteristics associated with attitudes towards the novel coronavirus disease in university-level Indian students. The findings indicated that there are some important differences in personality traits of people who take the COVID-19 virus as a serious threat and those who do not. Gaining an understanding of these associations between specific personality characteristics and COVID-19 management practices might assist in establishing more efficient public health actions helpful in mitigating the spread of this pandemic. For example, mental health professionals might promote programs that attempt to ameliorate some of the specific concerns (e.g., suicidal ideation, depression, anxiety, etc.) of those who view the virus as a serious threat. Further, these professionals might also create interventions that might change the attitudes of the virus deniers (e.g., appealing to their sense of humanity in that their behavior may adversely impact others around them, particularly children, the elderly, and other vulnerable individuals with whom they interact). In this fashion, deniers whose chief reason may be the protection of their own personal freedom, may maintain that attitude, but they may become aware that their personal responsibility also extends to the people around them. Considering these personality variations while designing preventive measures not only against COVID-19 but also other future health emergencies can be beneficial. The authors suggest further research on a much larger and much more diverse sample, including participants with varying ages, educational levels, and rural versus urban environments.

\section{Acknowledgements}

The authors wish to thank the administration at IIT Gandhinagar for their permission to conduct the present research.

\section{Conflicts of Interest}

The authors declare no conflicts of interest regarding the publication of this 
paper.

\section{References}

American Psychiatric Association (2013). Diagnostic and Statistical Manual of Mental Disorders (5th ed.). https://doi.org/10.1176/appi.books.9780890425596

Brezo, J., Paris, J., \& Turecki, G. (2006). Personality Traits as Correlates of Suicidal Ideation, Suicide Attempts, and Suicide Completions: A Systematic Review. Acta Psychiatrica Scandinavica, 113, 180-206. https://doi.org/10.1111/j.1600-0447.2005.00702.x

Carvalho, L. F., Pianowski, G., \& Gonçalves, A. P. (2020). Personality Differences and COVID-19: Are Extroversion and Conscientiousness Personality Traits Associated with Engagement with Containment Measures? Trends in Psychiatry and Psychotherapy, 42, 179-184. https://doi.org/10.1590/2237-6089-2020-0029

Coolidge, F. L. (2019). The Short-Form of the Coolidge Axis II Inventory (SCATI): Manual.

Coolidge, F. L., Segal, D. L., Benight, C. C., \& Danielian, J. (2004). The Predictive Power of Horney's Psychoanalytic Approach: An Empirical Study. American Journal of Psychoanalysis, 64, 363-374. https://doi.org/10.1007/s11231-004-4324-Z

Coolidge, F. L., Segal, D. L., Cahill, B. S., \& Simenson, J. T. (2010). Psychometric Properties of a Brief Inventory for the Screening of Personality Disorders: The SCATI. Psychology and Psychotherapy, 83, 395-405. https://doi.org/10.1348/147608310X486363

Fiala, J. A., Mansour, S. A., Matlock, S. E., \& Coolidge, F. L. (2020). Voter Perceptions of President Donald Trump's Personality Disorder Traits: Implications of Political Affiliation. Clinical Psychological Science, 8, 343-350.

https://doi.org/10.1177/2167702619885399

Furnham, A., \& MacRae, I. (2020). The Dark Side of Work Values. Current Psychology, 39, 1725-1731. https://doi.org/10.1007/s12144-018-9873-Z

Gralinski, L. E., \& Menachery, V. D. (2020). Return of the Coronavirus: 2019-nCoV. Viruses, 12, 135. https://doi.org/10.3390/v12020135

Lam, M. E. (2021). United by the Global COVID-19 Pandemic: Divided by Our Values and Viral Identities. Humanities and Social Sciences Communications, 8, 31. https://doi.org/10.1057/s41599-020-00679-5

Matias, T., Dominski, F. H., \& Marks, D. F. (2020). Human Needs in COVID-19 Isolation. Journal of Health Psychology, 25, 871-882. https://doi.org/10.1177/1359105320925149

Miguel, F. K., Machado, G. M., Pianowski, G., \& de Francisco Carvalho, L. (2021) Compliance with Containment Measures to the COVID-19 Pandemic over Time: Do Antisocial Traits Matter? Personality and Individual Differences, 168, Article ID: 110346. https://doi.org/10.1016/j.paid.2020.110346

Murphy, J., Vallières, F., Bentall, R. P., Shevlin, M., McBride, O., Hartman, T. K., McKay, R., Bennett, K., Mason, L., Gibson-Miller, J., Levita, L., Martinez, A. P., Stocks, T. V. A., Karatzias, T., \& Hyland, P. (2021) Psychological Characteristics Associated with COVID-19 Vaccine Hesitancy and Resistance in Ireland and the United Kingdom. Nature Communications, 12, 29. https://doi.org/10.1038/s41467-020-20226-9

O’Connell, K., Berluti, K., Rhoads, S. A., \& Marsh, A. A. (2021). Reduced Social Distancing Early in the COVID-19 Pandemic Is Associated with Antisocial Behaviors in an Online United States Sample. PLoS ONE, 16, e0244974.

https://doi.org/10.1371/journal.pone.0244974

Oosterhoff, B., \& Palmer, C. A. (2020). Attitudes and Psychological Factors Associated 
with News Monitoring, Social Distancing, Disinfecting, and Hoarding Behaviors among US Adolescents during the Coronavirus Disease 2019 Pandemic. JAMA Pediatrics, 174, 1184-1190. https://doi.org/10.1001/jamapediatrics.2020.1876

Petrosillo, N., Viceconte, G., Ergonul, O., Ippolito, G., \& Petersen, E. (2020). COVID-19, SARS and MERS: Are They Closely Related? Clinical Microbiology and Infection, 26, 729-734. https://doi.org/10.1016/j.cmi.2020.03.026

WHO World Health Organization (2020a). WHO Director-General's Opening Remarks at the Media Briefing on COVID-19 11 March 2020.

World Health Organization (2019). International Classification of Diseases (ICD-11).

World Health Organization (2020b). Coronavirus Disease (COVID-19) Pandemic.

World Health Organization (2020c). Coronavirus Disease (COVID-19) Advice for the Public. 